\title{
O campo das Habilidades Sociais no Brasil: Entrevista com Almir e Zilda Del Prette
}

\section{The field of Social Skills in Brazil: Interview with Almir and Zilda Del Prette}

\section{Carina Luiza Manolio* (in memoriam)}

Universidade Federal de São Carlos - UFSCar, São Carlos, SP, Brasil

\section{Bárbara Carvalho Ferreira**}

Universidade Federal de São Carlos - UFSCar, São Carlos, SP, Brasil

As relações interpessoais têm passado por grandes transformações nas últimas décadas e com isso o interesse em estudar esta temática vem aumentando consideralvemente. Várias áreas do conhecimento têm se proposto a investigar esse fenômeno, com destaque para o campo teórico-prático das Habilidades Sociais (HS).

No Brasil, são vários os pesquisadores interessados nesse campo de conhecimento, entre eles, Almir e Zilda Del Prette que, nos últimos anos vêm se dedicando com exclusividade à investigação desse tema. Esses pesquisadores foram os responsáveis pelas primeiras publicações sobre habilidades sociais em nosso país e ultimamente ambos têm incentivado a criação de grupos de pesquisadores em outras universidades. Uma das formas desse incentivo tem se concretizado por meio da coordenação do grupo de pesquisa Relações Interpessoais e Habilidades Sociais (RIHS http://www.rihs.ufscar.br) e do Grupo de Trabalho (GT) Relações Interpessoais e Competência Social, na Associação Nacional de Pesquisa e Pós-Graduação em Psicologia (ANPEPP http://www.anpepp.org.br).

Esse GT congrega vários grupos de pesquisa sobre habilidades sociais no Brasil. Os líderes desses grupos reúnem-se a cada dois anos em um evento organizado pela ANPEPP, no qual discutem e delimitam os diferentes objetos de pesquisa e planejam atividades de divulgação científica como congressos, simpósios, encontros, publicações em parceria para os dois anos seguintes a esse evento.

Todo esse movimento tem contribuído para que o Brasil seja o país da América Latina com maior número de publicações e pesquisadores na área de Habilidades Sociais. De acordo com o estudo bibliométrico sobre a produção científica desse campo teórico-prático das habilidades sociais na América Latina, de Nilsson, Suarez e Olaz (s.d.), o país é responsável por cerca de $72 \%$ dessa produção. 0 
estudo mostra também que, entre os autores com maior número de publicações, cinco deles são brasileiros e um argentino, sendo que Almir e Zilda Del Prette são os pesquisadores com maior número de trabalhos publicados no Brasil, o que já vinha sendo também apontado em outros estudos nacionais (BOLSONI-SILVA; DEL PRETTE; DEL PRETTE; FUMO; MANOLIO, BELLO; HAYASHI, 2009; MONTAGNER; BANDEIRA; DEL PRETTE, 2006; MURTA, 2005).

Apesar da alta produção na área de habilidades sociais no contexto brasileiro, foi na última década que se observou um maior avanço na literatura dessa área. Com isso, muitas questões ainda persistem e, com o objetivo de esclarecer alguns aspectos históricos, conceituais, metodológicos e práticos que permeiam o campo das Habilidades Sociais, foi realizada esta entrevista com os pesquisadores Almir e Zilda Del Prette. A entrevista foi dividida nas três seções que se seguem: (1) Histórico da área; (2) Aspectos teórico-práticos do THS; (3) O futuro do campo das HS no país.

\section{Histórico da Área}

1. Considerando o surgimento, por volta da década de 60 , do Treinamento de Habilidades Sociais (THS) na Europa e do Treinamento Assertivo na América do Norte, como esses movimentos influenciaram o desenvolvimento desta área no contexto brasileiro?

Almir. A divulgação sobre tais movimentos, nessa época, em nosso país, praticamente inexistiu. Havia alguma divulgação sobre o Treinamento Assertivo com a publicação, em 1978, do livro de Alberti e Emmons (1978) uma versão baseada na edição de 1973, revista pelos autores pouco tempo depois. Isso quer dizer que as informações que possuíamos já estavam defasadas em sua origem. A literatura sobre o Treinamento de Habilidades Sociais demorou cerca de 30 anos para se iniciar entre nós, quando a maioria dos teóricos já entendia a assertividade como uma subclasse de habilidades sociais. Mas ainda hoje, mesmo no Brasil, podem ser encontrados estudos que tomam o Treinamento das Habilidades Sociais como sucedâneo do Treinamento Assertivo, o que é historicamente um equívoco.

\section{Qual o histórico da produção científica no campo teórico- prático das Habilidades Sociais no Brasil?}

Almir. Com base nas publicações disponíveis, pode-se citar como pioneiro, um artigo de 1978 publicado pela revista Arquivos Brasileiros de Psicologia (DEL PRETTE, 1978), mas especificamente sobre habilidades sociais, o primeiro artigo ocorreu somente em 1996, na revista Psicologia: Reflexão e Crítica (DEL PRETTE; DEL PRETTE, 1996). No mesmo ano, surgiu um capítulo de livro de um grupo da ANPEPP (DEL PRETTE; DEL PRETTE, 1996) e, no ano seguinte, pela primeira vez apareceu um capítulo sobre habilidades 
sociais na coletânea da Associação Brasileira de Psicoterapia e Medicina Comportamental - ABPMC (DEL PRETTE; DEL PRETTE, 1997). Dois anos após, agora em 1999, foi publicado o primeiro livro sobre habilidades sociais no Brasil (DEL PRETTE; DEL PRETTE, 1999). Pode-se dizer que essas publicações lançaram as bases da divulgação desse campo entre nós. Nos anos seguintes, o número de publicações aumentou progressivamente, merecendo estudos de revisão, como por exemplo, Del Prette e Del Prette (2000); Mitsi, Silveira e Costa (2004); Murta (2005); Bolsoni-Silva, Del Prette, Del Prette, Montagner, Bandeira e Del Prette (2006); Fumo, Manolio, Bello e Hayashi (2009). Um estudo bibliométrico mais recente, realizado na Argentina (NILSSON; SUAREZ; OLAZ, s.d.) que analisa a produção da América Latina, destacou a contribuição brasileira como a mais vigorosa e trazendo novos elementos à história desse campo no Brasil.

\section{Quais as preocupações que nortearam os primeiros estudos no campo teórico-prático das habilidades sociais no país?}

Almir. As preocupações iniciais estavam associadas à formação de alunos-estagiários de Psicologia em diferentes lugares e às queixas de estudantes de Psicologia e de outros cursos sobre seus desempenhos interpessoais. Tais preocupações, inicialmente acadêmicas, possuíam uma dimensão clínica, levando-nos a realizar programas de habilidades sociais com alunos de graduação, especialmente em fases finais da graduação. Verificamos posteriormente que essas queixas também ocorriam em outros países e constituíam focos relevantes de pesquisa. Ao lado dessas preocupações com a formação do estudante de Psicologia, as revisões disponíveis (referidas na questão anterior) mostram uma predominância inicial (década de 80 ) de pesquisas voltadas para a construção de instrumentos e estudos de levantamento que somente mais tarde são secundadas por trabalhos de intervenção (ainda hoje incipientes) e, paralelamente, um aumento progressivo de estudos com diferentes tipos de população e com diferentes delineamentos de pesquisa. Especificamente em relação a trabalhos de intervenção, um primeiro estudo (quase-experimental, com grupo controle), conduzido no Brasil, avaliou os efeitos de um programa de habilidades sociais com alunos de Psicologia e foi publicado em 1978 (DEL PRETTE, 1978). Posteriormente, conduzimos vários outros programas, com diferentes objetivos, agora na comunidade. Um dos programas realizados com grupos na comunidade foi objeto de pesquisa de nossa dissertação de mestrado: eu identifiquei e analisei os procedimentos utilizados na intervenção (DEL PRETTE, 1982) e Zilda analisou o processo da intervenção (DEL PRETTE, 1983). 


\section{Atualmente, como tem se configurado, no Brasil, a produção científica na área?}

Zilda. No Brasil, são poucos os pesquisadores que se dedicam exclusivamente à temática das habilidades sociais. A maioria se dedica a essa temática em articulação com outros temas como transtornos psicológicos específicos, qualidade de vida, práticas parentais, relações interpessoais no trabalho, educação escolar (regular e especial), inteligência etc. Ao mesmo tempo em que isso mostra a potencialidade dessa área, indica também a necessidade de articular as direções de aprofundamento da produção científica. Um direcionamento de pesquisa importante e recente na área tem sido o investimento em procedimentos de observação direta, em situação natural e estruturada, para a avaliação e caracterização de habilidades sociais e uma preocupação com a validade convergente de diferentes indicadores de habilidades sociais. Outra preocupação recorrente é com o desenvolvimento de instrumentos e procedimentos para a avaliação e promoção de habilidades sociais em contexto de saúde e transtornos psicológicos, com desdobramentos para as práticas culturais, a qualidade de vida e as questões éticas da atuação psicológica nesse campo. No entanto, ainda não se tem uma revisão completa dos estudos mais recentes que poderiam sinalizar, de forma mais confiável, as tendências atuais. Falta, especialmente, uma revisão dos estudos que estão sendo conduzidos por meio de monografias, teses e dissertações. Estas, até onde temos conhecimento, em geral focalizam temáticas variadas, incluindo delineamentos experimentais e quase experimentais e investigam o impacto e o processo de programas de intervenção, bem como estratégias e recursos para a promoção de habilidades sociais. As revisões com base em artigos e capítulos já publicados ainda não dão conta dessa possível tendência.

\section{Quais as semelhanças e diferenças da produção científica do campo teórico-prático das Habilidades Sociais no Brasil e nos demais países (por exemplo, Estados Unidos, Argentina, Espanha)?}

Zilda. A principal diferença está possivelmente relacionada à tradição histórica. A produção científica nesse campo se iniciou realmente no Brasil nos anos 90 enquanto que nos outros países, especialmente nos Estados Unidos, remonta aos anos 50 e 60. Assim, enquanto no Brasil se verifica um interesse crescente e uma atualização constante na área, essa tendência não é tão forte nos demais países, especialmente na Espanha. A produção de Vicente Caballo na Espanha, da década de 80/90, não foi seguida de um movimento tão vigoroso como o que ocorreu no Brasil nos últimos 20 anos. Nos Estados Unidos, a produção na área permanece constante e há grupos produzindo e vitalizando a área, como por exemplo, os de 
Gresham (atualmente na Lousiana), de Merrell (California), de Bellack e Hersen (Pensilvania), de Curran e Monti (Rhode Island), de Liberman (Califórnia), de Conger e Conger (Indiana), de Matson (também de Louisiana, falecido em 2010, aos 78 anos).

\section{Aspectos teóricos e práticos do THS}

\section{Quais abordagens teóricas embasam o campo teórico- prático das Habilidades Sociais?}

Zilda. O interesse pelas relações interpessoais e pelas habilidades de relacionamento pode ser encontrado sob diferentes abordagens conceituais. No entanto, a produção consistente nessa área se ancora principalmente em três abordagens mais gerais: a Análise do Comportamento (AC), a abordagem Cognitiva (com uma multiplicidade de teorias) e a Teoria da Aprendizagem Social (Bandura). A produção científica sob perspectiva da Análise do Comportamento e, em particular, sob a aplicação dessa abordagem, constituiu um desenvolvimento inicial tão importante que alguns autores (p.e., O' DONOHUE; KRASNER, 1995) chegam a considerar que o movimento do THS vitalizou o desenvolvimento inicial da Terapia Comportamental. As abordagens cognitivas enfatizam os esquemas e processos encobertos do desempenho social, enquanto a Análise do Comportamento destaca o papel das contingências ambientais e da história de interação do indivíduo com o ambiente sobre as características abertas e encobertas do desempenho social. A teoria da aprendizagem social é usualmente associada ao campo das habilidades sociais por conta dos processos de aprendizagem por modelação, mas a versão mais recente das formulações teóricas de Bandura, a Teoria Social-Cognitiva, que recorre a processos cognitivos na explicação de relações entre processos comportamentais e ambientais, só recentemente vem sendo explorada no campo das habilidades sociais (ver, por exemplo, PEREZ, 2000; OLÁZ, 2009).

\section{O Treinamento de Habilidades Sociais (THS) pode ser caracterizado como método ou como uma técnica de intervenção?}

Almir. Alguns autores colocam o THS como um método ou uma técnica de intervenção. Pode-se questionar a noção de THS como técnica, uma vez que se trata de um conjunto de procedimentos de intervenção, onde são utilizadas várias técnicas, seja em contexto de grupo ou individual. Portanto, o mais coerente seria denominar método de intervenção.

\section{Quais os principais termos da área, suas definições e diferenças?}


Zilda. Considerando as bases conceituais que vêm se configurando nos últimos tempos, temos defendido três conceitos básicos da área: (a) desempenho social, que se refere a todo e qualquer comportamento que ocorre na interação entre pessoas; (b) habilidades sociais, que se refere às classes de comportamento social que contribuem para a competência social, ou seja, as classes que, quando utilizadas na interação social, apresentam alta probabilidade de produzir os resultados característicos da competência social; (c) competência social, que se refere à qualidade/funcionalidade do desempenho apresentado pelo indivíduo na interação social, ou seja, a uma avaliação desse desempenho com base em um conjunto de critérios. Conforme temos defendido ao longo de diversas publicações (DEL PRETTE; DEL PRETTE, 1999; DEL PRETTE; DEL PRETTE, 2001a; DEL PRETTE; DEL PRETTE, 2005), esses critérios contemplam simultaneamente uma dimensão instrumental (o que o indivíduo ganha na interação em termos imediatos) e ética (os ganhos para ambos os interlocutores e a para a relação entre eles, considerandose não apenas resultados imediatos mas o impacto em médio e longo prazo). Esses aspectos são fundamentais para se compreender a importância da competência social para a saúde, a qualidade de vida e o delineamento de práticas culturais mais compatíveis com a filosofia e a ideologia de uma profissão que deve estar preocupada com o bem estar e o desenvolvimento do ser humano.

\section{Quais os equívocos e críticas mais frequentes, em termos teóricos e práticos, ao campo das Habilidades Sociais.}

Zilda. Além de alguns aspectos já pontuados nas questões anteriores, há um equívoco recorrente em relação aos objetivos de programas de habilidades sociais. Alguns profissionais, seja por contato superficial com a área, seja por desinformação, entendem que o objetivo de um programa de habilidades sociais seria a promoção de padrões topográficos específicos de desempenho social. Em artigo recente (DEL PRETTE; DEL PRETTE, s.d.), buscamos esclarecer algumas características e objetivos dos programas de THS, mostrando o quanto essa compreensão é restrita, pelo menos dentro da perspectiva vivencial com a qual trabalhamos. Na referida publicação, destacamos, entre outras, as seguintes características dos programas vivenciais de THS: a ênfase na variabilidade comportamental (base para a flexibilidade e ajuste do desempenho a diferentes demandas), a exposição e o desenvolvimento da sensibilidade às contingências (mais do que a regras pré-fixadas de comportamento), o ensino da análise funcional e da automonitoria (também condição para a flexibilidade e criatividade do comportamento social), o foco nas habilidades de processo (um objetivo intermediário e condição importante para o alcance dos demais objetivos). No artigo, também questionamos o uso do termo 
"treinamento", de algum modo consagrado na literatura, mas pouco adequado a um programa que contempla as características e objetivos acima referidos e que não seriam condizentes com a conotação comum da palavra treinamento.

\section{Dentre os vários cuidados necessários para a condução de um programa de intervenção em Habilidades Sociais, quais os principais a serem adotados?}

Almir. Existem pelo menos dois cuidados que merecem atenção especial. Tanto no processo terapêutico como no educacional, em contexto de grupo ou não, o primeiro, refere-se ao preparo do terapeuta. A condução de um programa de habilidades sociais requer domínio de várias técnicas de intervenção e arranjos de diferentes procedimentos. Outro cuidado refere-se ao aspecto ético. Com muita frequência, as queixas dos clientes que sugerem necessidade de THS, podem se referir a indicadores de dependência do cliente a um ou mais significantes como pais, namorado(a), cônjuge etc. Nesses casos, não se trata de desenvolver apenas um repertório de contracontrole que, com alta probabilidade, pode inverter o quadro dominante-dominado. Mais do que isso, o cuidado ético deve orientar a intervenção para uma nova dimensão nos relacionamentos, substituindo, tanto quanto possível, o ganha-perde para uma dimensão "ganha-ganha" (DEL PRETTE; DEL PRETTE, s.d.). Outros cuidados, de natureza técnica, são facilmente localizados nos manuais, tais como o planejamento adequado do programa com base em diagnóstico multimodal, avaliações continuas, planejamento de generalização etc.

\section{Quais os métodos mais utilizados para: (a) avaliação do repertório de habilidades sociais; (b) promoção das HS; (c) avaliação dos programas de intervenção?}

Almir. A avaliação de habilidades sociais requer um delineamento multi-informante e multimodal em termos de diferentes informantes, procedimentos e tipos de indicadores (por exemplo, relato e observação direta). A promoção de habilidades sociais em geral requer um conjunto articulado de procedimentos e recursos que vão dos instrucionais aos vivenciais, aqui se incluindo o uso de recursos multimídia, leituras, tarefas de casa, monitoramento e tutoria etc. Já a avaliação de programas requer procedimentos e instrumentos que permitam verificar os efeitos imediatos e mediatos da intervenção sobre o repertório de comportamentos dos participantes e também o impacto dessas mudanças sobre as relações com as demais pessoas, a qualidade de vida e o desenvolvimento pessoal ou profissional. Há ainda as avaliações de processo, que são importantes para o aperfeiçoamento dos programas em termos de efetividade e em 
termos de sua validade social (por exemplo, a avaliação da integridade da intervenção, da satisfação do consumidor etc.).

12. O método vivencial, presente nos programas de intervenção de Habilidades Sociais no Brasil, também é utilizado em outros países?

Zilda. O método vivencial, tal como o utilizamos no Brasil, não é comum em outros países, onde há nitidamente uma ênfase instrucional. O máximo de experiência mais direta com o desempenho interpessoal, encontrado nesses programas, são os procedimentos de desempenho de papéis (role-playing), de modelação e de ensaio comportamental. Temos informação de que o método vivencial vem sendo utilizado na Argentina (seguindo o modelo brasileiro), inclusive com pesquisa que compara programas com e sem esse método e que mostraram melhores resultados com o método vivencial.

\section{Além do método vivencial, quais outros recursos, inclusive} tecnológicos, podem ser utilizados nestes tipos de programas? Zilda. Os recursos multimídia (vídeos, vinhetas, estórias contadas, livretos etc.) são recursos potencialmente efetivos e importantes de serem explorados em programas de habilidades sociais. Em estudo recente no Brasil, as vinhetas do IMHSC-Del-Prette (DEL PRETTE; DEL PRETTE, 2005) foram utilizadas como base de um programa de habilidades sociais para crianças, com resultados bastante promissores (LOPES, 2009). Outros recursos desenvolvidos pelo grupo de pesquisa vêm sendo explorados, como vídeos (CÔMODO, 2008), estorinhas (SILVA, 2008) e jogos (FREITAS; LEMMI, 2009) As possibilidades são ilimitadas e há alguns estudos em andamento explorando jogos de tabuleiro e games, o que pode vitalizar também essa área em nosso meio.

\section{O futuro do campo das HS no país}

14. Dentre as várias questões e temas estudados no campo teórico-prático das Habilidades Sociais, quais apresentam produção significativa e quais precisariam ainda de mais investigações adicionais?

Zilda. Na reunião deste ano do nosso Grupo de Trabalho na Associação Nacional de Pesquisa e Pós-Graduação em Psicologia (ANPEPP), discutimos essa questão e resumimos, no Relatório Final (DEL PRETTE; DEL PRETTE, 2010), um conjunto de encaminhamentos relevantes para a pesquisa no Brasil, que deveria nortear os futuros estudos, especialmente de pós-graduação e dos grupos de pesquisa ativos. Esses encaminhamentos foram reunidos sob três eixos:

Avaliação. Já existem vários instrumentos de autoinforme produzidos no Brasil e adequados à nossa cultura, produzidos pelo nosso grupo 
da UFSCar e também pelos demais grupos, como os liderados pela colega Eliane Falcone (UERJ) e Alessandra Bolsoni-Silva (UNESPBauru). Isso não quer dizer que novos instrumentos sejam dispensáveis, mas sim, que se pode direcionar os esforços para a adaptação e padronização de novos instrumentos agora voltados para diferentes tipos de população disfuncionais (por exemplo, para cegos via Braille, para deficientes mentais e outros tipos de problemas específicos). Identifica-se, igualmente a necessidade de instrumentos/procedimentos observacionais, como os explorados mais intensivamente pelo grupo liderado pela colega Marina Bandeira, da UFSJ (Minas Gerais) e por nosso grupo da UFSCar bem como de escalas breves, uma tendência no campo da saúde, que vem sendo explorada pelo grupo liderado pela colega Sonia Loureiro, da USP de Ribeirão Preto.

Intervenção. Já dispomos de vários estudos mostrando a efetividade dos programas de habilidades sociais, ainda que a maioria seja recente e pouco esteja publicado. No entanto, se carece de uma avaliação mais detalhada desses programas que busque identificar os ingredientes críticos dos "pacotes de intervenção" que caracterizam esses programas. Isso implica adotar delineamentos mais refinados de controle de validade interna (por exemplo, controle nãotratamento, validação social e placebo fragmentado). Também se carece de estudos de seguimento (follow-up), com avaliação de generalização para outras situações e manutenção no tempo, bem como de maior investimento na construção e avaliação de recursos para uso nesses programas.

Questões sociais culturais e psicossociais. Entende-se que é necessário maior investimento em estudos de natureza conceitual, crítica e ética sobre o significado e a contribuição dos programas de habilidades sociais e sua possível inserção e contribuição em políticas de saúde, qualidade de vida e desenvolvimento em geral. Tais estudos também deveriam focalizar o papel desses programas em práticas parentais, comunitárias, de treinamento e educativas, visando o empoderamento das pessoas e mudanças no cenário de direitos humanos e cidadania.

Em termos de futuro, entendemos que o movimento das habilidades sociais no Brasil tem alta probabilidade de se manter e até mesmo aumentar em visibilidade e importância, considerando que essa temática representa uma resposta às necessidades profissionais da Psicologia e de áreas afins, com aplicação bastante conspícua aos problemas de relacionamento interpessoal e intergrupal na sociedade contemporânea. Entendemos também que a produção científica brasileira, que até agora vem se concentrando em periódicos nacionais, tem condições de se expandir e ampliar o intercâmbio com os pesquisadores de outros países, o que certamente se reverterá em novas questões e refinamento metodológico. 


\section{O que é a ANPEPP? Como funciona o GT-ANPEPP? Quantos e quais são os grupos de pesquisa em habilidades sociais no Brasil?}

Zilda. A ANPEPP é a Associação de Pesquisa e Pós-Graduação em Psicologia no Brasil, criada com o objetivo principal de congregar os pesquisadores e programas de pós-graduação, definir políticas de produção de conhecimento e encaminhar soluções para os desafios da pesquisa em Psicologia. Como os pesquisadores investigam diferentes objetos, foram criados os Grupos de Trabalho (GTs) que permitem um intercâmbio mais intensivo entre pesquisadores interessados em um mesmo tema ou objeto de investigação. Inicialmente, havia uma reunião anual da ANPEPP que depois, devido ao crescimento de participantes, passou a ser bienal. O GT Relações interpessoais e Competência Social é constituído pelos líderes dos núcleos de pesquisa em habilidades sociais e inclui pesquisadores de 10 diferentes instituições. Alguns desses núcleos focalizam temáticas bem específicas, como pode ser visto na página web: http: //www.rihs. ufscar. br/ equipe-1/nucleosrihs-e-gt-anpepp.

Este GT começou a funcionar em 2002 e, em cada reunião, são feitas propostas de atividades coletivas de pesquisa e/ou divulgação, por exemplo, o I e o II Seminário Internacional de Habilidades Sociais e os dois livros já produzidos pelo grupo (DEL PRETTE; DEL PRETTE, 2001b; DEL PRETTE; DEL PRETTE, 2003). O conjunto das propostas e encaminhamentos deste quinto encontro do GT foi reunido no Relatório Final (DEL PRETTE; DEL PRETTE, 2010), disponível no site da ANPEPP e do Grupo RIHS. Neste encontro de 2010, houve uma participação mais ativa dos doutorandos de pós-graduação que constituem, a nosso ver, a base da continuidade do grupo e do movimento das habilidades sociais no Brasil. Nesse sentido, podemos identificar "nichos" de investigação e de prática que poderiam ser assumidos por novos grupos de pesquisa, visando o desenvolvimento da área no Brasil.

\section{Considerações Finais}

Considerando o teor das respostas concedidas pelos professores Almir Del Prette e Zilda Del Prette a esta entrevista, pode-se ter um panorama de aspectos históricos, teóricos, práticos e de questões futuras para o campo teórico-prático das Habilidades Sociais no Brasil. Os entrevistados apresentaram reflexões sobre o histórico da área neste país (fazendo um paralelo com outros países), tanto no que se refere ao desenvolvimento da área como às questões iniciais que motivaram os primeiros estudos com esta temática no contexto brasileiro.

Além das preocupações que direcionaram pesquisas iniciais da área, Almir e Zilda apontaram questões atuais de investigação, discutidas 
inclusive no Grupo de Trabalho na ANPEPP-2010, que podem nortear novos estudos relacionados às questões conceituais, sociais, culturais e psicossociais concomitantemente à continuidade da produção científica empírica da avaliação e intervenção sobre o repertório de habilidades sociais de diferentes clientelas.

Um outro aspecto que merece destaque na entrevista é a preocupação de Almir e Zilda com questões teóricas e práticas que permeiam a área. Assim, por meio de esclarecimentos sobre as diferentes abordagens teóricas que embasam o campo das habilidades sociais (controvérsias teóricas que podem confundir 0 leitor); da explicação e diferenciação dos principais termos da área; da elucidação dos equívocos conceituais e práticos mais frequentes; da apresentação dos cuidados necessários para a condução de programas de intervenção em HS; dos métodos mais utilizados para avaliação do repertório de HS, avaliação de programas de intervenção e promoção de HS; e da ilustração de recursos que podem ser utilizados em intervenções nesta área (vivências, recursos multimídia, jogos, estorinhas, vídeos). Pode-se concluir que a presente entrevista traz contribuições preciosas para a contextualização e o desenvolvimento do campo teórico-prático das Habilidades Sociais no Brasil.

No que se refere aos novos estudos na área tendo em vista a perspectiva de aumento no número de pesquisadores que realiza doutorado no campo das habilidades sociais, pode-se prever uma nova fase quanto aos objetos e métodos de pesquisa e também em relação à divulgação desses estudos em periódicos e livros, nacionais e do exterior. A participação dos doutorandos no GT-ANPEPP de 2010, ainda que concentrando orientandos dos dois pesquisadores entrevistados, representou um importante passo nessa direção. Espera-se que essa oportunidade e seus desdobramentos sejam ampliados nos próximos simpósios.

\section{Referências Bibliográficas}

ALBERTI, R. E.; EMMON, M. L. Comportamento assertivo: Um guia de auto-expressão. Belo Horizonte: Interlivros, 1978.

BOLSONI-SILVA, A. T.; DEL PRETTE, Z. A. P.; DEL PRETTE, G.; MONTAGNER, A. R.; BANDEIRA, M.; DEL PRETTE, A. Habilidades sociais no Brasil: Uma análise dos estudos publicados em periódicos. In: BANDEIRA, M.; DEL PRETTE, Z. A. P.; DEL PRETTE, A. (Orgs.). Estudos sobre habilidades sociais e relacionamento interpessoal. São Paulo: Casa do Psicólogo, 2006, p.17-45. CÔMODO, C. N. Aprendizagem de habilidades sociais para préescolares: Recursos de vídeo. 2008. Trabalho de Conclusão de 
Curso. Graduação em Psicologia, Universidade Federal de São Carlos, São Carlos.

DEL PRETTE, A. O treino assertivo na formação do psicólogo. Arquivos Brasileiros de Psicologia Aplicada, Rio de Janeiro, v. 30, p. 53-55, 1978.

Treinamento comportamental em grupo junto à população não clínica de baixa renda: Uma análise descritiva de procedimento. 1982. Dissertação (Mestrado em Psicologia Clínica) Programa de Pós-Graduação em Psicologia, Pontifícia Universidade Católica de Campinas, Campinas.

DEL PRETTE, Z. A. P. Uma análise descritiva de um programa de treinamento comportamental em grupo junto à população não clínica de baixa renda. 1983. Dissertação (Mestrado em Psicologia Social). Universidade Federal da Paraíba, João Pessoa.

DEL PRETTE, A.; DEL PRETTE, Z. A. P. Habilidades sociais e construção de conhecimento em contexto escolar. In: ZAMIGNANI, D. R. (Org.). Sobre comportamento e cognição: A aplicação da Análise do Comportamento e da Terapia Cognitivo-Comportamental no hospital geral e nos transtornos psiquiátricos. São Paulo: AirBytes, 1997, p. 234-250.

- Psicologia das relações interpessoais: Vivências para o trabalho em grupo. Petrópolis: Vozes, $2001 a$.

Psicologia escolar e educacional, saúde e qualidade de vida: Explorando fronteiras. Campinas: Editora Alínea, 2001b.

. Habilidades sociais, desenvolvimento e aprendizagem: Questões conceituais, avaliação e intervenção. Campinas: Editora Alínea, 2003.

Programa vivencial de habilidades sociais: características sob a perspectiva da Análise do Comportamento. Submetido para publicação na Coletânea Comportamento e Cognição. s.d.

DEL PRETTE, Z. A. P.; DEL PRETTE, A. Habilidades sociais: Uma área em desenvolvimento. Psicologia: Reflexão e Crítica, Porto Alegre, v. 9, n. 2, p. 287-389, 1996.

Habilidades envolvidas na atuação do psicólogo escolar/educacional. In WECHSLER, S. M. (Org.). Psicologia Escolar: Pesquisa, formação e prática. Campinas: Alínea, 1996, p. 139-156.

- Psicologia das Habilidades Sociais: Terapia, educação e trabalho. 4. ed. Petrópolis: Ed. Vozes, 1999.

- Treinamento em habilidades sociais: Panorama geral da área. In: HAASE, V. G.; ROTHE-NEVES, R.; KÄPPLER, C.; TEODORO, M. L. M.; WOOD, G. M. O. (Eds.). Psicologia do Desenvolvimento: Contribuições Interdisciplinares. Belo Horizonte: Health, 2000, p. 249-264. 
Petrópolis: Ed. Vozes, 2005a.

Habilidades sociais na infância: Teoria e prática.

Sistema Multimídia de Habilidades Sociais para

Crianças (SMHSC-Del-Prette). São Paulo: Casa do Psicólogo, 2005b.

. Relatório final ANPEPP 2010: GT-53 Relações Interpessoais e

Competência Social. Disponível em:

<http://www.rihs. ufscar.br/armazenagem/pdf/relatorio-anpepp-

2010>. Acesso em: 9 jul. 2010.

FUMO, V. M. S.; MANOLIO, C. L.; BELLO, S.; HAYASHI, M. C. P. I. Produção científica em habilidades sociais: estudo bibliométrico. Revista Brasileira de Terapia Comportamental e Cognitiva, Campinas, v. 11, n. 2, p. 246-266, 2009.

FREITAS, L. C.; LEMMI, R. C. A. Elaboração de um recurso educativo para identificação e expressão de emoções. Paidéia, Ribeirão Preto, v. 19, p. 403-405, 2009.

LOPES, D.C. Recursos multimídia na promoção de habilidades sociais com crianças com dificuldades de aprendizagem. 2009. Dissertação (Mestrado em Educação Especial). Programa de PósGraduação em Educação Especial. Universidade Federal de São Carlos, São Carlos.

MITSI, C. A.; SILVEIRA, J. M.; COSTA, C. E. Treinamento de Habilidades Sociais no tratamento do transtorno obsessivo compulsivo: Um levantamento bibliográfico. Revista Brasileira de Terapia Comportamental e Cognitiva, Campinas, v. 6, n. 1, p. 4959, 2004.

MURTA, S. G. Aplicações do Treinamento em Habilidades Sociais: Análise da Produção Nacional. Psicologia: Reflexão e Crítica, Porto Alegre, v. 18, n. 2, p. 283-291, 2005.

NILSSON, N. S. P.; SUAREZ, A. B.; OLÁZ, F. O. Estudio bibliométrico de las publicaciones científicas sobre el área de habilidades sociales en américa latina. Artigo disponibilizado pelos autores. s.d.

O' DONOHUE, W.; KRASNER, L. Psychological skills training. In: O'Donohue, W.; Krasner, L. (Eds.). Handbook of Psychological Skills Training. London: Allyn and Bacon, 1995, p. 1-19

OLÁZ, F. O. Contribuições da Teoria Social-Cognitiva de Bandura para o Treinamento de Habilidades Sociais. In: DEL PRETTE, Z. A. P.; DEL PRETTE, A. (Orgs.). Psicologia das Habilidades Sociais: Diversidade teórica e suas implicações. Petrópolis: Ed. Vozes, 2009, p. 109-148.

SILVA, A. P. C. Manual para pais: Atividades lúdicas para o ensino de habilidades sociais a pré-escolares. 2008. Trabalho de Conclusão de Curso (Graduação em Psicologia) - Universidade Federal de São Carlos, São Carlos. 


\section{Endereço para correspondência}

Família de Carina Luiza Manolio

Rua Carlos Gomes, 907, Centro, CEP 15900-000, Taquaritinga-SP, Brasil

Endereço eletrônico: mjmanolio@yahoo.com.br

Bárbara Carvalho Ferreira

Avenida Professor Luis Augusto de Oliveira, 289, apt.09, Vila Celina, CEP 13566340, São Carlos-SP, Brasil

Endereço eletrônico: babipocos@yahoo.com.br

Recebido em: 23/07/2010

Aceito para publicação em: 28/07/2010

Acompanhamento do processo editorial: Adriana Benevides Soares

\section{Notas}

* Psicóloga graduada pela Pontifícia Universidade Católica de Campinas/PUCCampinas, Mestre em Educação Especial pela UFScar; Doutoranda em Psicologia pela Universidade Federal de São Carlos - UFSCar. Carina faleceu em 24/01/2011. **Psicóloga graduada pela Universidade Federal de São J oão Del Rei - UFS J, Mestre em Educação Especial pela UFScar; Doutoranda em Psicologia pela Universidade Federal de São Carlos - UFSCar. 